David Holmes (D)

\title{
Quasi-compactness of Néron models, and an application to torsion points
}

Received: 2 August 2016 / Accepted: 15 September 2016

Published online: 29 September 2016

\begin{abstract}
We prove that Néron models of Jacobians of generically-smooth nodal curves over bases of arbitrary dimension are quasi-compact (hence of finite type) whenever they exist. We give a simple application to the orders of torsion subgroups of Jacobians over number fields.
\end{abstract}

\section{Introduction}

If $S$ is a regular scheme, $U \subseteq S$ is dense open, and $A / U$ is an abelian scheme, then a Néron model for $A / S$ can be defined by exactly the same universal property as in the case where $S$ has dimension 1. We do not impose a priori that the Néron model should be of finite type, and we allow the Néron model to be an algebraic space (rather than just a scheme) for flexibility. We investigate this in detail in [8], giving necessary and sufficient conditions for the existence of Néron models in the case of Jacobians of nodal curves. In this short note we prove that, in the setting of Jacobians of nodal curves, a Néron model is quasi-compact (and hence of finite type) over $S$ whenever one exists (Theorem 2.1).

Note that Néron models of non-proper algebraic groups (such as $\mathbb{G}_{m}$ ) need not be quasi-compact. Moreover, in $[2, \S 10.1,11]$ an example (due to Oesterlé) is given of a Néron model over a dedekind scheme all of whose fibres are quasicompact, but which is not itself quasi-compact. Thus in general the question of quasi-compactness of Néron models can be somewhat delicate.

We give an application to controlling the orders of torsion points on abelian varieties. Recall that by [4] the uniform boundedness conjecture for Jacobians of curves is equivalent to the same conjecture for all abelian varieties. By considering the universal case, the uniform boundedness conjecture is equivalent to

Conjecture 1.1. (Uniform boundedness conjecture) Let $U$ be a scheme of finite type over $\mathbb{Q}$ and $C / U$ a smooth proper curve. Let $\sigma \in J(U)$ be a section of the Jacobian. Let $d \geq 1$ be an integer. Then there exists an integer $B$ such that for every point $u \in U(\overline{\mathbb{Q}})$ with $[\kappa(u): \mathbb{Q}] \leq d$, the order of the point $\sigma(u) \in J_{u}$ is either infinite or at most $B$.

D. Holmes ( $\varangle$ ): Universiteit Leiden, Leiden, Netherlands. e-mail: 26davidholmes26@gmail.com

Mathematics Subject Classification: Primary 11G10; Secondary 14K30 14 K05 
We will show (Corollary 3.5) that this conjecture holds if there exists a compactification $U \hookrightarrow S$ over which $C$ has a proper regular model with at-worst nodal singularities, and over which $J$ has a Néron model. The first two conditions are relatively mild, especially since it is actually enough to work up to alterations (cf. [5]), but the assumption that a Néron model should exist is very strong. For the sake of those readers unfamiliar with the theory developed in [8], we mention

Corollary 1.2. (Immediate from Corollary 3.5) Let $S / \mathbb{Q}$ be a smooth proper variety, let $Z \longmapsto S$ be a closed subvariety which is smooth over $\mathbb{Q}$. Let $C / S$ be a family of nodal curves which is smooth outside $Z$. Write $U=S \backslash Z$ and let $\sigma \in J(U)$ be any section of the family of Jacobians. Then for every $d \geq 1$ there exists an integer $B$ such that for every point $u \in U(\overline{\mathbb{Q}})$ with $[\kappa(u): \mathbb{Q}] \leq d$, the order of the point $\sigma(u) \in J_{u}$ is either infinite or at most $B$.

This can be viewed as a common generalisation (in the case of Jacobians) of the following standard results:

Proposition 1.3. (Proper smooth base) Let $S / \mathbb{Q}$ be a proper smooth variety and $A / S$ an abelian scheme. Then for every $d \geq 1$ there exists an integer $B$ such that for every point $s \in S(\overline{\mathbb{Q}})$ and every $a \in A_{S}(\overline{\mathbb{Q}})$ with $[\kappa(a): \mathbb{Q}] \leq d$, the order of the point $a$ is either infinite or at most $B$.

Proposition 1.4. (Base of dimension 1) Let $S / \mathbb{Q}$ be a smooth variety of dimension 1 , and let $A / S$ be an abelian scheme. Let $\sigma \in A(S)$ be any section. Then for every $d \geq 1$ there exists an integer $B$ such that for every point $s \in S(\overline{\mathbb{Q}})$ with $[\kappa(s): \mathbb{Q}] \leq d$, the order of the point $\sigma(s) \in A_{s}$ is either infinite or at most $B$.

Proposition 1.3 is easy to prove and is well-known; Proposition 1.3 follows for example from [11]. Other examples are given in Sect. 3.1.

Neither Corollary 1.2 nor the stronger form Corollary 3.5 is very useful for proving that the full uniform boundedness conjecture holds for a given family of Jacobians; a Néron model exists for the tautological curve over a family of curves $C / S$ if and only if $C / S$ is of compact type, in which case the uniform boundedness conjecture is in any case easy (cf. Corollary 3.4).

In [9] we consider related questions where we replace torsion points with points of 'small height'. However, the results of [loc.cit] are more restrictive since we need to make an additional technical positivity assumption on the base $S$ of our family. The techniques used to prove those results are very different from what we do here, though [loc.cit] does use the quasi-compactness result proven in this paper.

Many thanks to Owen Biesel, Maarten Derickx, Bas Edixhoven, Wojciech Gajda, Ariyan Javanpeykar, Robin de Jong and Pierre Parent for helpful comments and discussions. The author would also like to thank the anonymous referee for a very rapid report suggesting a number of improvements. Finally, the author is very grateful to an anonymous referee at Crelle for pointing out a gaping hole in an earlier proof given in [8] of the quasi-compactness of such Néron models. 


\section{The Néron model is of finite type}

Before giving the proof of our main theorem we briefly recall some definitions we need from $[7,8]$. In what follows, $S$ is a scheme. Details can be found in the above references.

A nodal curve over $S$ is a proper, flat, finitely presented morphism, all of whose geometric fibres are reduced, connected, of dimension 1 , and have at worst ordinary double point singularities. If $C / S$ is a nodal curve then we write $\mathrm{Pic}_{C / S}^{[0]}$ for the subspace of $\mathrm{Pic}_{C / S}$ consisting of line bundles which have total degree zero on every fibre. If $s \in S$ is a point then a non-degenerate trait through $s$ is a morphism $f: T \rightarrow S$ from the spectrum $T$ of a discrete valuation ring, sending the closed point of $T$ to $s$, and such that $f^{*} C$ is smooth over the generic point of $T$.

We say a nodal curve $C / S$ is quasisplit if the morphism $\operatorname{Sing}(C / S) \rightarrow S$ is an immersion Zariski-locally on the source (for example, a disjoint union of closed immersions), and if for every field-valued fibre $C_{k}$ of $C / S$, every irreducible component of $C_{k}$ is geometrically irreducible.

Suppose we are given $C / S$ a quasisplit nodal curve and $s \in S$ a point. Then we write $\Gamma_{s}$ for the dual graph of $C / S$ at $s$-this makes sense because $C / S$ is quasisplit and so all the singular points are rational points, and all the irreducible components are geometrically irreducible. Assume that $C / S$ is smooth over a schematically dense open of $S$. If we are also given a non-smooth point $c$ in the fibre over $s$, then there exists an element $\alpha \in \mathcal{O}_{S, s}$ and an isomorphism of completed étale local rings (after choosing compatible geometric points lying over $c$ and $s$ )

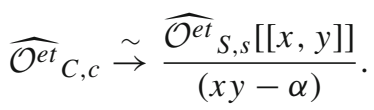

This element $\alpha$ is not unique, but the ideal it generates in $\mathcal{O}_{S, s}$ is unique. We label the edge of the graph $\Gamma_{s}$ corresponding to $c$ with the ideal $\alpha \mathcal{O}_{S, s}$. In this way the edges of $\Gamma_{s}$ can be labelled by monogenic ideals of $\mathcal{O}_{S, s}$.

If $\eta$ is another point of $S$ with $s \in \overline{\{\eta\}}$ then we get a specialisation map

$$
\text { sp: } \Gamma_{s} \rightarrow \Gamma_{\eta}
$$

on the dual graphs, which contracts exactly those edges in $\Gamma_{s}$ whose labels generate the unit ideal in $\mathcal{O}_{S, \eta}$. If an edge $e$ of $\Gamma_{s}$ has label $\ell$, then the label on the corresponding edge of $\Gamma_{\eta}$ is given by $\ell \mathcal{O}_{S, \eta}$.

Theorem 2.1. Let $S$ be a regular scheme, $U \hookrightarrow S$ a dense open subscheme, and $C \rightarrow S$ a nodal curve which is smooth over $U$ and with $C$ regular. Write $J$ for the jacobian of $C_{U} \rightarrow U$. Assume that $J$ admits a Néron model $N$ over $S$. Then $N$ is of finite type over $S$.

Proof. The Néron model is smooth and hence locally of finite type; we need to prove that it is quasi-compact over $S$.

Step 1: Preliminary reductions. 
The existence of the Néron model implies by [8] that the Néron model coincides canonically with the quotient of $\mathrm{Pic}_{C / S}^{[0]}$ by the closure of the unit section $\bar{e}$, and the latter is flat (even étale) over $S$.

Given an integer $n \in \mathbb{N}$, we write $\operatorname{Pic}_{C / S}^{|-| \leq n}$ for the subfunctor of $\mathrm{Pic}_{C / S}^{[0]}$ consisting of line bundles which on every fibre of $C / S$ have all partial degrees bounded in absolute value by $n$ and of total degree zero. Clearly $\operatorname{Pic}_{C / S}^{|-| \leq n}$ is of finite type over $S$ for every $n$, and we have a composite map $\mathrm{Pic}_{C / S}^{|-| \leq n} \rightarrow \mathrm{Pic}_{C / S}^{[0]} \rightarrow N$. If we can show that this composite is surjective for some $n$ then we are done.

We may assume without loss of generality that $S$ is noetherian, since every regular scheme is locally noetherian, and quasi-compactness is local on the target. Then we are done if we can make a constructible function $\underline{n}: S \rightarrow \mathbb{N}$ such that for all $s \in S$, the composite $\mathrm{Pic}_{C_{s} / s}^{|-| \leq \underline{n}(s)} \rightarrow N_{s}$ is surjective.

Fix a point $s_{0} \in S$. We are done if we can find a non-empty open subset $V \hookrightarrow \overline{\left\{s_{0}\right\}}$ and an integer $n \in \mathbb{N}$ such that for all $s \in V$, the composite $\mathrm{Pic}_{C_{s} / s}^{|-| \leq n} \rightarrow N_{s}$ is surjective.

Step 2: Graphs and test curves.

After perhaps replacing $S$ by an étale cover, we may assume that $C / S$ is quasisplit. By [7, lemma 6.3] there exists an open subset $V \hookrightarrow \overline{\left\{s_{0}\right\}}$ such that for all $s \in V$, the specialisation map sp: $\Gamma_{s} \rightarrow \Gamma_{s_{0}}$ is an isomorphism on the underlying graphs.

Claim: After shrinking $V$, there exists an integer $m$ such that for all $s \in V$ there exists a non-degenerate trait $f_{S}: T_{S} \rightarrow S$ through s such that for every edge e of $\Gamma_{s}$, we have

$$
\left|\operatorname{ord}_{T_{s}} f_{s}^{*} \operatorname{label}(e)\right| \leq m .
$$

Two things remain to complete the proof: we must prove the claim, and deduce the theorem from the claim.

Step 3: Proving the claim. Replacing $S$ by an étale cover (and $s_{0}$ by a point lying over it) we can arrange the following:

- $\quad S$ is affine (say $S=\operatorname{Spec} A$ );

- There exist generators $a_{1}, \ldots, a_{n}$ of the prime ideal $s_{0} \subseteq A$ such that for each $0 \leq i \leq n$, the closed subscheme $V\left(a_{1}, \ldots, a_{i}\right) \subseteq S$ is connected and regular, is of codimension $i$, and is not contained in the closed subscheme of $S$ over which $C$ is not smooth.

Let $X$ be the closed subscheme of $S$ cut out by $a_{1} \ldots, a_{n-1}$. Then $X$ is regular and connected, and the image $x$ of $s_{0}$ in $X$ is of codimension 1, cut out by the image $a \in \mathcal{O}_{X}(X)$ of $a_{n}$.

For each edge $e$ of $\Gamma$, write label $(e) \in \mathcal{O}_{S, x}$ for its label. After shrinking $S$, we may assume that all these labels lie in $\mathcal{O}_{S}(S)$. The images of the labels on $X$ lie in $\mathcal{O}_{X}(X)$, and so after perhaps shrinking $X$ we can assume that every label is equal (up to multiplication by a unit in $\mathcal{O}_{X}(X)$ ) to some power of $a$.

Set $V:=V(a) \subseteq X$. Fix $v \in V$, and write $\mathfrak{m}_{v}$ for the maximal ideal of the local ring at $v$. By the regularity assumptions we see that $a \in \mathfrak{m}_{v}$ 
and $a \notin \mathfrak{m}_{v}^{2}$. Hence we can find a set of elements $r_{1}, \ldots, r_{d} \in \mathfrak{m}_{v}$ such that the image of $a, r_{1}, \ldots, r_{d}$ in $\mathfrak{m}_{v} / \mathfrak{m}_{v}^{2}$ are a basis as an $\mathcal{O}_{X, v} / \mathfrak{m}_{v}$-vector space. Then define $T_{v}$ to be the subscheme $V\left(r_{1}, \ldots, r_{d}\right)$ of $\operatorname{Spec} \mathcal{O}_{X, v}$, and it is clear that $a$ pulls back to a uniformiser in the trait $T_{v}$, so we have $\operatorname{ord}_{T_{v}} f_{v}^{*} a=1$.

Step 4: Deducing the theorem from the claim.

We know the formation of $\mathrm{Pic}_{C / S}^{[0]}$ commutes with base change. As remarked above, $\bar{e}$ is flat over $S$ by our assumption that the Néron model exists. Because of this flatness, it follows that formation of the closure of the unit section also commutes with base-change.

Now let us fix for each $s \in V$ a non-degenerate trait $f_{S}: T_{S} \rightarrow S$ through $s$ as in the statement of the claim. Then the thicknesses of the singularities of $C_{T_{s}} / T_{s}$ are bounded in absolute value by $m$ as $s$ runs over $V$ (note that the graphs of the central fibres are the same for all $s \in V$ ). By $[6, \S 2]$ the multidegrees of points in the closure of the unit section can be described purely in terms of the combinatorics of the dual graph. Since there are only finitely many possibilities for this dual graph, we find an integer $n$ such that for all $s \in V$, the composite

$$
\operatorname{Pic}_{C_{T_{S}} / T_{S}}^{|-| \leq n} \rightarrow \frac{\operatorname{Pic}_{C_{T_{s}} / T_{s}}^{[0]}}{\bar{e}}=N_{S}
$$

is surjective.

\section{Consequences for torsion points}

Given a field $k$, by a variety over $k$ we mean a separated $k$-scheme of finite type. We fix a number field $K$ and an algebraic closure $\bar{K}$ of $K$. We write $\kappa(p)$ for the residue field of a point $p$. If $X / K$ is a variety and $d \in \mathbb{Z}_{\geq 1}$ then we write $X(\bar{K})^{\leq d}$ for the set of $x \in X(\bar{K})$ with $[\kappa(x): K] \leq d$.

Definition 3.1. Let $S / K$ be a variety, and $A / S$ an abelian scheme.

1. We say the uniform boundedness conjecture holds for $A / S$ if for all $d \in \mathbb{Z}_{\geq 1}$ there exists $B \in \mathbb{Z}$ such that for all $s \in S(\bar{K})$ and all torsion points $p \in A(\bar{K})_{s}^{\leq d}$, the point $p$ has order at most $B$.

2. Given a section $\sigma \in A(S)$, we say the uniform boundedness conjecture holds for the pair $(A / S, \sigma)$ if for all $d \in \mathbb{Z}_{\geq 1}$ there exists $B \in \mathbb{Z}$ such that for all $p \in S(\bar{K})^{\leq d}$, the point $\sigma(p)$ either has infinite order or has order at most $B$.

If the uniform boundedness conjecture holds for $A / S$ then it clearly holds for the pair $(A / S, \sigma)$ for all $\sigma$. By considering the case of the universal PPAV, we deduce that if the uniform boundedness conjecture holds for all pairs $(A / S, \sigma)$ then the uniform boundedness conjecture itself (Conjecture 1.1) holds. 
Lemma 3.2. Fix an integer $g \geq 0$ and a prime power $q$. Then there exists an integer $b=b(g, q)$ such that for every connected commutative finite-type group scheme $G / \mathbb{F}_{q}$ of dimension $g$ we have $\# G\left(\mathbb{F}_{q}\right) \leq b$.

Proof. Since $\mathbb{F}_{q}$ is perfect, the scheme $G^{\text {red }}$ is a subgroup scheme and contains all the field-valued points, so we may assume $G$ is reduced and hence smooth. Again using that $\mathbb{F}_{q}$ is perfect, we can apply Chevalley's theorem to write an extension

$$
1 \rightarrow T \times U \rightarrow G \rightarrow A \rightarrow 1
$$

where $A$ is abelian, $T$ is a torus and $U$ is connected and unipotent. We know $U$ is isomorphic (as a scheme over $\mathbb{F}_{q}$ ) to $\mathbb{A}_{\mathbb{F}_{q}}^{n}$ for some $n \leq g$ by [10, Remark A.3], so we have uniform bounds on the sizes of $A\left(\mathbb{F}_{q}\right), T\left(\mathbb{F}_{q}\right)$ and $U\left(\mathbb{F}_{q}\right)$, from which the result is immediate.

Theorem 3.3. Let $S$ be a proper scheme over $K$, let $A / S$ be a finite-type commutative group algebraic space with connected geometric fibres, and let $\sigma \in A(S)$ be a section. Then for every integer $d>0$ there exists a bound $B$ such that for all $s \in S(\bar{K})^{\leq d}$, the point $\sigma_{s}$ is either of infinite order or is torsion of order at most $B$.

Proof. To simplify the notation we treat the case $K=\mathbb{Q}$ and $d=1$; the general case is very similar. We begin by observing that we can 'spread out' the proper scheme $S$, the group algebraic space $A$ and the section $\sigma$ over $\mathbb{Z}[1 / N]$ for some sufficiently divisible integer $N$ - we use the same letters for the 'spread out' objects. Let $p$ be a prime number not dividing $N$, and let $b=b(\operatorname{dim} A / S, p)$ be the bound from Lemma 3.2, noting that a finite-type group algebraic space over a field is a group scheme by [1]. Suppose we are given $s \in S(K)$ (with unique extension $\bar{s} \in S(\mathbb{Z}[1 / N])$ since $S$ is proper over $\mathbb{Z}[1 / N])$ such that $\sigma_{s}$ is torsion. For some $n$ the point $\tau:=p^{n} \sigma_{s}$ is torsion of order prime to $p$. Then the subgroup algebraic space of $A_{\bar{s}}$ generated by $\tau$ is étale over $\mathbb{Z}_{p}$, and so the order of the torsion point $\tau$ is bounded above by $b$ (independent of $s \in S(K)$ ). In this way we control the prime-to- $p$ part of the order, and by considering another prime $l$ we can control the whole order.

In the case of abelian varieties, we immediately obtain

Corollary 3.4. Let $S$ be a proper scheme over $K, U \subseteq S$ dense open, $A / U$ an abelian scheme, and $\sigma \in A(U)$ a section. Suppose there exists a finite-type commutative group algebraic space with connected fibres $\bar{A} / S$ extending $A$, such that $\sigma$ extends to $\bar{A}(S)$. Then the uniform boundedness conjecture holds for $(A / S, \sigma)$.

By considering the diagonal section of the tautological family of abelian varieties $A \times{ }_{S} A$ over $A$, we deduce that the uniform boundedness conjecture holds for an abelian scheme over a proper smooth base, i.e. when $U=S$ (Proposition 1.4).

The proper smooth case is rather trivial, and it is not a priori clear how to construct interesting examples for Corollary 3.4. In the case of Jacobians of nodal curves the situation becomes much better; we understand exactly when Néron models exist by [8], and by Theorem 2.1 we know that they are always of finite 
type, so any section of the jacobian family extends to the identity component of the Néron model after taking some finite multiple (which is harmless for the arguments). We obtain

Corollary 3.5. Let $S$ be a proper scheme over $K$ and $C / S$ a regular family of nodal curves, smooth over some dense open $U \subseteq S$. Let $\sigma \in J(U)$ be a section of the jacobian family. If $J / U$ admits a Néron model over $S$ then the uniform boundedness conjecture holds for $(J / U, \sigma)$.

\subsection{Examples}

We finish by giving some examples where this result can be applied. Recall from [8] that the jacobian $J$ admits a Néron model if and only if the curve $C / S$ is aligned; in other words that for all geometric points $s$ of $S$, the labelled dual graph $\Gamma_{s}$ described in Sect. 2 has the property that

for every circuit $\gamma$ in $\Gamma_{s}$, and for every pair of edges $e_{1}, e_{2}$ appearing in $\gamma$, the labels of $e_{1}$ and $e_{2}$ satisfy a multiplicative relation of the form

$$
\operatorname{label}\left(e_{1}\right)^{n_{1}}=\operatorname{label}\left(e_{2}\right)^{n_{2}}
$$

for some positive integers $n_{1}$ and $n_{2}$.

Thus we see that $J$ admits a Néron model over $S$ (and so Corollary 3.5 applies) if any of the following hold (note that in each case $S$ must be proper in order to apply Corollary 3.5).

1. $S$ has dimension 1 (Proposition 1.4), so all labels are powers of a uniformiser in the base (this recovers a weaker version of a theorem of Silverman [11] and Tate [12] on heights in families of varieties);

2. $C / S$ is of compact type (all dual graphs are trees); then the Néron model is an abelian scheme (cf. Proposition 1.3);

3. All dual graphs of $C / S$ are treelike (i.e. a tree with some loops added). The Néron model need not be proper, but its component groups will be trivial;

4. The complement of $U$ in $S$ (with reduced scheme structure) is smooth over $K$, cf. corollary 1.2. Here we do not need to assume that $C$ is regular, since in this case we can resolve singularities without disturbing alignment.

In Example 3 the assumption that $C$ be regular is crucial, as we can illustrate with elliptic curves. Let $\mathcal{M}_{1,1} / K$ be the moduli stack ${ }^{1}$ of stable 1-pointed curves of genus 1 , and let $\mathcal{M}_{1,2}$ be the universal stable 'pointed elliptic curve'. Now $\mathcal{M}_{1,1}$ has treelike-fibers so by Example 3 we can apply Corollary 3.5 to the regular curve $\mathcal{M}_{1,2}$ over $\mathcal{M}_{1,1}$ (we could alternatively use that $\mathcal{M}_{1,1}$ has dimension 1 ). However, if we want to re-prove the uniform boundedness conjecture for elliptic curves we would need to apply Corollary 3.5 to the universal stable curve $\mathcal{M}_{1,3}$ over $\mathcal{M}_{1,2}$. Note that $\mathcal{M}_{1,3}$ can be obtained by blowing up $X:=\mathcal{M}_{1,2} \times \mathcal{M}_{1,1} \mathcal{M}_{1,2}$. Now

\footnotetext{
1 All our results work just as well when the base is an algebraic stack.
} 
$X / \mathcal{M}_{1,2}$ does have treelike fibres, but when we resolve the singularities this breaks down, and indeed $\mathcal{M}_{1,3}$ over $\mathcal{M}_{1,2}$ does not have treelike fibres and its jacobian does not admit a Néron model. Similar considerations can be used to see that our results cannot be used to recover those of [3].

Open Access This article is distributed under the terms of the Creative Commons Attribution 4.0 International License (http://creativecommons.org/licenses/by/4.0/), which permits unrestricted use, distribution, and reproduction in any medium, provided you give appropriate credit to the original author(s) and the source, provide a link to the Creative Commons license, and indicate if changes were made.

\section{References}

[1] Artin, M.: Algebraization of Formal Moduli. I. In: Spencer, D.C., Iyanaga S. (eds.) Global Analysis: Papers in Honor of K. Kodaira (PMS-29). Princeton University Press, pp. 21-71 (1969). http://www.jstor.org/stable/j.ctt13x10qw

[2] Bosch, S., Lütkebohmert, W., Raynaud, M.: Néron Models. Springer, Berlin (1990)

[3] Cadoret, A., Tamagawa, A.: Uniform boundedness of p-primary torsion of abelian schemes. Invent. Math. 188(1), 83-125 (2012)

[4] Cadoret, A., Tamagawa, A.: Note on torsion conjecture. In: Geometric and Differential Galois Theories, Volume 27 of Séminar Congress, pp. 57-68. Society Mathematics France, Paris (2013)

[5] de Jong, A.J.: Smoothness, semi-stability and alterations. Inst. Hautes Études Sci. Publ. Math. 83, 51-93 (1996)

[6] Edixhoven, B.: On Néron models, divisors and modular curves. J. Ramanujan Math. Soc. 13(2), 157-194 (1998)

[7] Holmes, D.: A Néron model of the universal jacobian. http://arxiv.org/abs/1412.2243 (2014)

[8] Holmes, D.: Néron models of jacobians over base schemes of dimension greater than 1. J. Reine Angew. Math. http://arxiv.org/abs/1402.0647 (2014)

[9] Holmes, D.: Torsion points and height jumping in higher-dimensional families of abelian varieties. arXiv preprint http://arxiv.org/abs/1604.04563v1 (2016)

[10] Kambayashi, T., Miyanishi, M., Takeuchi, M.: Unipotent Algebraic Groups. Lecture Notes in Mathematics, vol. 414. Springer, Berlin (1974)

[11] Silverman, J.H.: Heights and the specialization map for families of abelian varieties. J. Reine Angew. Math. 342, 197-211 (1983)

[12] Tate, J.: Variation of the canonical height of a point depending on a parameter. Am. J. Math. 105(1), 287-294 (1983) 